\title{
Study of Tamil Particles in Wider and Deeper Contexts
}

\author{
K. Karunakaran* and R. Krishnan
}

University of Malaya, Kuala Lumpur, karuna@um.edu.my, krishnamoorthykarunakaran32@gmail.com

\begin{abstract}
Particles can be found in noun morphological forms and verb morphological forms to denote meaning in wider and deeper contexts. The aim of this study is to identify all those particles in modern Tamil and classify them based on their occurrence in morphological forms and phrases and explain their contextual/functional significance and explain their functional importance in achieving adequacy and coherency in language structure and language use with reference to modern standard Tamil focusing on competence and performance of the language users.
\end{abstract}

Keywords: Accent, Appropriateness, Coherency, Cohesiveness, Competence, Inflected Forms, Morphological Forms, Pitch, Syntactico-semantic Relation, Tone

\section{Introduction}

Particles found in languages are considered as inflected forms. They have functional and contextual significance in language structure to achieve cohesiveness. Particles enter into grammar as they are added to major grammatical categories such as nouns and verbs, that is in noun morphological forms and verb morphological forms. All these forms are used in phrases and sentences in an order to denote meaning in wider and deeper contexts. So, the aim of this study is to: i) identify all those particles in modern Tamil and classify them based on their occurrence in morphological forms and phrases and explain their contextual/functional significance, ii) to identify those particles which participate mostly in discourse, and explain their functional importance in achieving adequacy and coherency in language structure and language use with reference to modern standard Tamil.

This study making use of theoretical framework tries to explain the role of particles in grammar and formation of discourse for different purpose in modern Tamil. Particles participate in different contexts and help to achieve appropriateness, adequacy and coherency. This kind of achievement is quite needed for effective communication. Hence, this study tries to focus on two aspects of language structure and language use namely i) competence and ii) performance of the language users or those who participate in communicative contexts , situations etc.

\section{Frame Work Used}

There are particles as shown in the following structures:

i) atuvarai, patittavarai, inRuvarai ......

ii) ta:yaippo:la, ennaivita, tampiyaippaRRi,...

iii) ya:ro:, eppatiyo:, eppolto: , ....

iv) ya:ra:vatu, eppatiya:vatu, enke:ya:vatu, eppoluta:vatu , ....

v) munpin, me:lumki:lum , ....

vi) vantirunta: lum, vanta: lunku:ta, unka Lutaiyatuta: na, eppatiya: na: lum......

vii) a:na:l, me:lum, inime:lum, me:nme:lum,

viii) innumkuRippittucconna:l, curunkkau:Rin,

ix) oruva:ru, ore:yatiya:ka .....

${ }^{*}$ Author for correspondence 


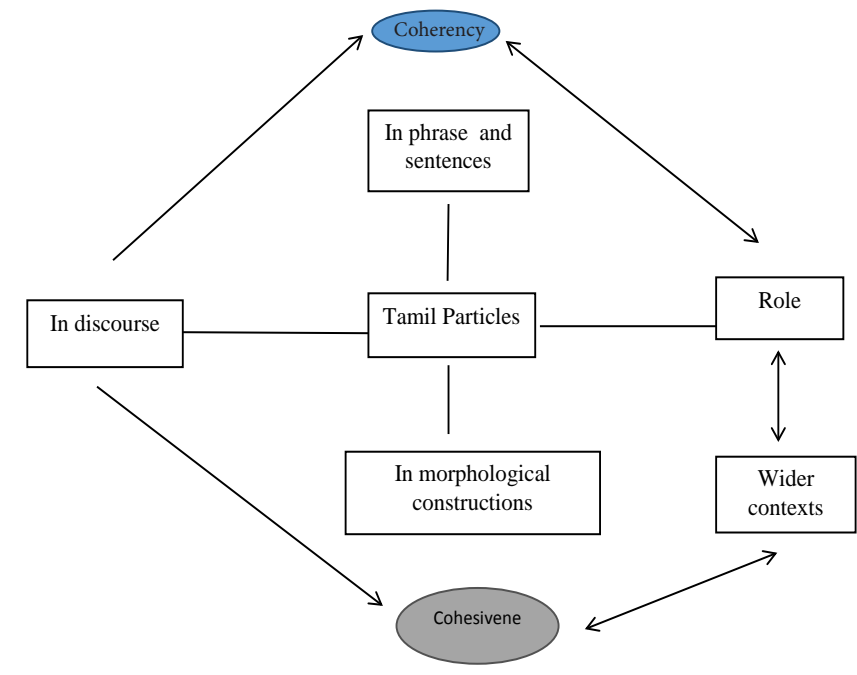

x) mika, mikavum ....., mikamika ......

xi) etuepptiya:na:lum ......

\section{Achieving Cohesiveness and Coherence in Discourse in Modern Tamil - An Assessment with the Help of the Analysis}

Cohesiveness is concerned with the structural part of language used to convey a particular communication using appropriate linguistic forms pertaining to a particular context. Structure includes not only the basic word forms but also grammatical forms such as phrases, sentences and beyond which participate in a discourse. When such forms occur in a suitable way and conditioned order the communicator is able to express himself or herself as expected or in a more effective and appropriate way. In other words, those who read or happened to listen to one's written or spoken discourse he or she is able to comprehend and understand fully or partially. That means there exists not only a relevant grammatical relationship between or among the usages but also cohesiveness in structure to explain a particular contextual meaning. So, cohesiveness is an act of not only competence but also relates to performance with reference to comprehension or understanding. Even one can say that cohesiveness leads to more coherency or paves way for better understanding of a communication. This kind of dependency of cohesiveness and coherence makes a discourse more meaningful and fluent.

The aspect of coherence though the focus is on meaning conveyed it relates the structure with the content of the communication and so there is a correlation between coherency and cohesiveness from the point of view of comprehension. When structures are chosen to fit into a discourse (in the discourse formation) due importance is given to the key concepts or ideas one wants to express. So, lexical items, compounds or short phrases are chosen first. All these items then enter into larger constructions not only by using different types of sentences but also extra elements which are needed to connect the sentences wherever necessary. This is the reason why we use particles (one or more depending upon the need) along with phrases and sentences even though they are not part of sentence structures. So, we find certain initiators at the beginning of a discourse or sentences in a discourse followed but some of the continuators in order to maintain the continuity in a discourse. We also make use of connectors to relate the thoughts expressed through sentences at appropriate places. Hence, the role of initiators, continuators and connectors becomes more essential in order to achieve the required cohesiveness in expression and coherency in thought.

Though language structures play a vital role in communication there are extra linguistic features such as pitch, tone, accent, etc. including the body language matters for better expression and comprehension. In order to describe the cohesiveness and coherency in a discourse one needs to analyze the syntactico-semantic relationship found among the sentences related to one another which found place in a discourse.

\section{Discourse Particles and Their Function}

\subsection{The Role of Initiators in Achieving Adequate Structural Cohesiveness}

Initiators are conditioned by socio cultural contexts or situations also. Address forms in Tamil are not only used as initiators but also can occur as closures in a sentence or discourse.

\section{Example:}

e:nTa: tampi:! n:i avaniTam mikavum kavanama:ka iru. e:nenRa:l avan mikavum polla:tavanTa:

( hi! younger brother, you have to be very careful with him because he is a very dangerous fellow.)

This kind of usage of particles brings out the closeness between the speaker and hearer on one hand and that of caution or advice given to the hearer on the other. So the 
content is explained by using expressions needed to show the closeness as well as the significance of the caution given through the particular expression.

There is another type of initiators namely $a T a, a T a T a$ : used while putting forward the thoughts which have significance with reference to a particular discourse. Example,

aTa/aTaTa:avanvantuTTa:npa:rtta:ya: ( what a surprise, you see he has come (a tone of excitement/ astonishment))

There are expressions which are used just for the purpose of initiating a conversation in order to communicate something. Sometimes they act as attention seekers. In Tamil particles like appo: (when), pa:ruńka(you see), inta (this), ata:n(that only) etc. function like this. These particles are not part of any sentence, they do not convey any particular meaning but their occurrences have functional significance as they can make the hearer or listener to get involved in the concerned communication and respond.

There is yet another set of particles which function as continuators in a discourse. These continuators not only help to have continuity in communication but also make the participants to take turns and communicate their responses or views. In other words, it encourages the participant's role in communication. Example, particles like piRaku(then), aTuttu(next), ataRkuppiRaku(after that), atumaTTumilla:mal(not only that), me:lum(further), innumcollappo:na:l (to add to it) etc.

The following are some of the relevant features in the study of particles and their occurrence.

(i) Use of particles as initiators in discourse

(ii) Use of agreement rules

(iii) Use of continuators to maintain free flow of communication as in conversations etc.

(iv) Use of connectors like a:na:1, enave:, atana:1 etc. as in $\mathrm{S} 1+$ a:na:l (etc.) + S2

(v) Use of continuators like appuRam, piRaku, enna, vantu, vantuTTu, cari, nallatu, etc.

\section{Particles Which Occur in a Discourse}

There are at least five types of occurrence of particles with various grammatical constituents in Modern Tamil discourse. They are as follows:

\subsection{As Sentence Connectors (Initiators)}

Structure: Single Forms

Function : As sentence initiators but functionally connecting the previous sentence with it (the following one).

\subsection{As Sentence Connectors (Initiators with more than One Form)}

Structure: (i) One Particle + a grammatical Form

(ii) A grammatical Form + particle

(iii) More than one particle

Function: Used as sentence initiators (or paragraph initiators) but functionally they serve as connectors.

\subsection{As Continuators in Discourse}

Structure:

(i) Use of only one form

(ii) Use of more than one form

Function:

(i) To continue the discourse with cohesion and coherence needed for the context

(ii) To maintain continuity of expression in order to achieve cohesiveness explicitly

\subsection{As Sentence Connectors using more than One Particle}

Structure:

(i) Particle $1+$ Particle 2

(ii) Particle $1+$ Particle $2+$ Particle 3 etc.

Function: To maintain the needed cohesiveness in between sentences

\subsection{As Sentence Continuators and Initiators}

Structure: Short phrases or grammatical forms

Function: To maintain continuity of discourse and increase its readability and fluency

\subsection{As Sentence Initiators in the Context of Addressing}

(a) Structure: Address forms like Te:y, e:n Ta:, e:n Ti: ( attention markers or callers) 
Function : To initiate a conversation or to draw one's attention to a particular activity etc.

Example,

Te:yińkakoñcamva:ye:n! ( hei! please come here for a while)

e:mpa: (ennappa:) (hello young man) (used as an address term)

ennappa: eppa Tiyirukke ? (hei, young man how are you!)

(b) Structure: As sentence continuators Use of particles like ata:vatu (that is ), piRaku (later), appuRam (what next), etc.

Example :

piRaku, eppo: vanti:nka ? ennaceyti? (ok, when did you come? what news? / anything special?)

\subsection{As Sentence or Discourse Closures/ Terminators}

Form : Reference / Address forms

Use of particles and grammatical forms like Ta:, Ti:, Te: (attention markers), nallatu ( that's fine), caricari (ok), pa:rppo:m ( see you later), as closures.

Function: When making closing remarks or to close a conversation, to sum up the proceedings, etc.

Example :

cari Ta: ....oruva:Ru nnaTantumuTintatu.... pa:rppo:m eppaTippo:kiRatuenRu.

(ok my friend, somehow everything is over now. let us see how it works.)

Oruvakaiyil ca:ma:Litta:yiRRu. eppaTi ppo:kiRatu enRu pa:rppo:m.

(somehow we managed to do it. let us see how it works.)

\section{Description with Examples and Contexts}

\subsection{Type 1}

- $\quad$ toTakkattiliruntu inRuvarai avaruTaiyapo:kkaip poRuttukkoNTe:n. ini, enna:l poRuttukkoLLa muTiya:tu.( From the beginning till date I was passionate with him. I don't think I can be like that anymore.)

- intacceyalmuRai payanuLLatumaTTumanRi, a:RRal mikkatuńku:Ta. atana:l, intacceyalmuRai pinpaRRappaTTatu. (This approach was not only a useful one, but also an efficient one. Therefore, this approach was followed).

\subsection{Type 2}

- $\quad$ naTantavaiyella:m avvaLavuciRappa:ka amaiyavillaienpatil aiyamillai. Etu eppaTiya:yinum nammuTaiya muyaRcikaL toTarave:NTum enpate: anaivaruTaiya viruppam. ( whatever has happened so far were not that good. whatever the case may be everyone wants that our efforts should continue.)

- $\quad$ ni:ńkaL koTuttanu:l paTikka viRuviRuppa:ka irukkiRatu, a:na:lum katai o:TTattil a:ńka:ńke: koñcam taTuma:RRamum ka:NappaTukinRatu. ( The book you gave me is quite exciting to read. However, there are a few problems in the free flow of the story.)

\subsection{Type 3}

Use of ata:vatu (that is)

- avarkaL na:nkoTuttave:laiyaiccarivaracceytum uTikkavillaienpatu ennuTaiya karuttu. (They didn't complete the work which I entrusted to them in a proper way, that is my opinion.) e:n? ennanaTantatu? (why? what has happened?) ata:vatu, avarkaL na:n connava:Ru entaoru oluńkumuRaiyaiyum pinpaRRave: illai. (That is, they did not follow at all any systematic way as I instructed.)

There are more than one particle added one after the other in a phrase in order to convey additional meanings to make the discourse more cohesive and coherent in their structure and function.

Example :

ini + a:vatu >iniya:vatu or ini + me:l + a:vatu >inime:la:vatu (at least here after or from now on)

\subsection{Type 4}

Use of appaTiyenRa:1 ( if that is so )

Example: 
- atu carivara:tu enRu na:n ninaikkiRe:n. (I think it won't be suitable for this purpose.) appaTiyenRa:l, anta muRaiya:l namakku entappalanum e:RpaTa:ta:? ( In that case do you think that there will not be any benefit to us by using this method.)

\subsection{Type 5}

- oruva:Ra:ka avarkaLuTaiya muyaRcikaL veRRiyaTaintana, atana:1 anaivarukkum mananiRaivu e:RpaTTatu. (at last they were successful in their attempts, so everyone was quite satisfied.)

- eppaTiyo: antaccikkalai oruva:Ru cama:LittuviTTo:m. pa:rppo:m, ennanaTakkavuLLatuenRu. (somehow we were able to solve that problem in a way / in one way or the other. Let us see what is going to happen.)

\section{Cohesiveness and Sentence Structure}

Though there are a variety of compound and complex sentences in Tamil, sometimes particles like a:na:l (but), atana:1 (therefore ), enave:, a:kave: (so), etc.. are needed to connect two or more full sentences in order to maintain or to achieve the required cohesiveness between the connected sentences through which better coherency could be achieved.

Example:

na:n ne:RRuma:lai oru tirumaNavila:viRkuppo:yiru kkave:NTum ( yesterday I should have gone to attend a marriage reception) enakkuvetirpa:ra:malvoruve:lai vantuviTTatu ( unexpectedly I had to attend to an urgent work) atana:l, na:n nikalccikuppo:kavillai ( therefore, I did not attend the function)

These three sentences have meanings of their own but when they are put together in the form of a brief discourse there is a need to have connectors to make it more cohesive. So, we add particles a:na:l, atana:l etc. as connectors. This kind of addition makes it not only more cohesive in structure but also helps to make the syntactico-semantic interpretation correctly in order to understand the meaning.

\section{References}

1. Agesthialingom S. Colliyal-peyariyal-vinaiyiyal. Chennai: Manivasagar Pathippagam, 1998.

2. Alonso L. 'The SenSem Project: Syntactico- Semantic Annotation of Sentences in Spanish, In Nicolos, N. Kalina, B. Galia, A. \&Ruslan, M. (eds.), Recent Advances in Natural Language Processing IV. New York: John Benjamin Publishing Co, 2007.

3. Arokianathan S. Clitics in Modern Tamil. Pondicherry: PILC, 1980.

4. Huddleston R. Pullum G. A Student's Introduction to English Grammar. London: CUP, 2006.

5. Karunakaran K. A Simplified Grammar of Tamil. Chennai: Kumaran Pathippagam, 2000.

6. Karunakaran K. Krishnan R. Study of Particles in Modern Malaysian Tamil. Chennai: IITS, 2013.

7. Lehmann T. A Grammar of Modern Tamil. Pondicherry: PILC, 1991.

8. Nuhman M.A. A Tippa Tait Tamil Ilakka Nam. Tiruchirappalli: Adaiyalam, 2007. 